\title{
Severe Bandemia Is Not Associated With Increased Risk for Adverse Events in General Pediatric Emergency Department Patients
}

Daniel Najafali ${ }^{1}$, Noorvir Kaur ${ }^{1}$, Ikram Afridi ${ }^{1}$, Norhan Abdalla ${ }^{1}$, Leenah Afridi ${ }^{1}$, Iana Sahadzic ${ }^{1}$, Julianna Solomon ${ }^{1}$, Isha Yardi ${ }^{1}$, Quincy K. Tran ${ }^{1}$

1. Research Associate Program in Emergency Medicine and Critical Care, University of Maryland School of Medicine, Baltimore, USA

Corresponding author: Daniel Najafali, najafalid@gmail.com
Review began 01/21/2021 Review ended 02/08/2021 Published 02/12/2021

\section{(c) Copyright 2021}

Najafali et al. This is an open access article distributed under the terms of the Creative Commons Attribution License CC-BY 4.0., which permits unrestricted use, distribution, and reproduction in any medium, provided the original author and source are credited.

\begin{abstract}
Introduction: The presence of band cells > 10\% of the total white blood cell (WBC) count ("bandemia”) is often used as an indicator of serious bacterial illness (SBI). Results from studies of bandemia as a predictor of SBI were conflicting and little is known about the relationship between severe bandemia (SB) and clinical outcomes from SBI in children. We hypothesized that SB (band level $>20 \%$ ) is not associated with adverse outcomes in an emergency department (ED) pediatric population.
\end{abstract}

Methods: Medical records from children between the ages of two months and 18 years with SB who presented to a tertiary referral regional hospital were studied. Outcomes were categorized as severe adverse events (SAEs) or moderate adverse events (MAEs). Multivariate logistic regressions were used to assess the association between SB and outcomes.

Results: We analyzed 102 patients. Mean age (standard deviation, SD) was 5.25 (0.5) years, 18 (18\%) had MAE, 21 (21\%) had SAE, and no patients died. Mean band levels were similar between groups: no adverse events 28 (10) vs. SAE 31 (9) vs. MAE 27 (8), p=0.64. Multivariate logistic regressions showed SB was not associated with any adverse events (odds ratio (OR) 1.04, 95\% confidence interval (CI) 0.9-1.1, p=0.27). Nonnormal X-ray (XR) (OR 17, 95\% CI 3.3-90, p<0.001) was associated with MAE, while non-normal computerized tomography (CT) scan (OR 15.4, 95\% CI 2.2-100+, p=0.002) was associated with SAE.

Conclusion: SB was not associated with higher odds of adverse events among the general ED pediatric population. Clinicians should base their clinical judgment on the overall context of history, physical examinations, and other laboratory and imaging data.

Categories: Emergency Medicine, Pediatrics

Keywords: adverse outcome, bacterial illness, bandemia, general pediatric emergency department patients, severe bandemia

\section{Introduction}

Among the pediatric population in the United States, 50,000 cases of severe sepsis occur annually, a number that has been on the rise for a condition that carries a mortality rate of approximately 10\% [1]. In 1992, the Society of Critical Care Medicine designated a band cell concentration of more than $10 \%$ of the total peripheral white blood cell (WBC) count as one of the criteria for systemic inflammatory response syndrome [2]. Since then, many clinicians have considered an elevated band cell level as a surrogate for serious bacterial illness (SBI).

Bandemia was associated with bloodstream infection in the adult patient population [3]. In the pediatric emergency medicine literature, sustained controversy questions whether a band cell level greater than $10 \%$ of the WBC count truly indicates SBI. Jaskiewicz et al., writing in 1994, and Schnadower et al., publishing in 2010, claimed that pediatric patients with bandemia greater than $10 \%$ were at risk of SBI [4,5]. In contrast, other author groups in the past reported no association between bandemia greater than $10 \%$ and SBI [6-8]. Kuppermann et al. suggested that bandemia at $13 \%$ did not differentiate between pediatric patients with culture-proven or laboratory-proven bacterial infections and those with viral infections [8].

Most published studies considered a band level of $10 \%$ as the threshold for bacterial infection. There is scant evidence in the pediatric emergency medicine literature about the association between severe bandemia (SB) ( $>20 \%$ band cells) and clinical outcomes. We hypothesized that SB is not associated with an increased risk of adverse events and outcomes. This article was previously posted to the medRxiv preprint server on January 4, 2021.

\section{Materials And Methods}




\section{Study design}

We conducted a retrospective cohort study using medical records from a single pediatric emergency department (ED) at an urban, regional tertiary referral level one pediatric trauma center. The study was approved by the hospital's institutional review board.

\section{Patient population and data collection}

We created a convenience cohort by first identifying patients who were two months to 18 years of age when they were brought to the ED for evaluation between January and July 2008. All patients during the study period within the aforementioned age range were included. We selected this date because our regional pediatric hospital ceased to perform differential cell count on all patients' blood samples. Starting on August 1, 2008, differential cell count, and band levels, were only performed by orders of physicians who suspected infection in their patients. As a result, blood samples with bands after August 1, 2008 were most likely performed on patients with high suspicion for infection.

SB was defined as band cell levels $\geqslant 20$ (constituting $20 \%$ of the total peripheral WBC count) [9]. Any imaging study (computerized tomography, CT, scan or X-ray (XR) films) interpreted by attending radiologists as suggesting an infectious process would be considered "non-normal" imaging studies.

An investigator who was not blind to the study hypothesis abstracted the data into a standardized form using an Excel spreadsheet (Microsoft Corporation, Redmond, WA). Data were subsequently prepared by other investigators who were blind to the study hypothesis prior to analysis.

\section{Outcomes}

The primary outcome in this study was a severe adverse event (SAE) associated with bacterial illness. SAEs were defined as in-hospital death, hospital admission from the index ED visit or within seven days, returning to the ED within seven days, bacteremia (blood culture showing > 10,000 colony-forming units per milliliter), or positive cerebrospinal fluid (CSF, WBC count > 10 cells per high-power field, positive gram stain).

The secondary outcome was any moderate adverse event (MAE) associated with bacterial illness. These events were defined as positive urinalysis (urinary WBC count >100,000 cells/high-power field, positive nitrite or leukocyte esterase) and bacteria-positive stool cultures. The other outcome was any adverse event (AAE), which is a combination of SAEs and MAEs.

\section{Data analysis}

We first performed descriptive analyses to categorize patients in each subgroup. Continuous variables were assessed for normal distribution by the Ryan-Joiner test. Normally-distributed-data were expressed as mean (standard deviation, SD) and compared via the Student's t-test. Chi-square tests were used to compare groups of categorical data, while analysis of variance (ANOVA) with Holm-Sidak post-hoc tests, were used to compare means between groups of normally-distributed-continuous-data.

We also performed multivariable logistic regressions to assess the association between clinical factors and outcomes (SAE, MAE, or AAE). Statistical analyses were performed with Sigma Plot version 13.0 (Systat Software Inc., San Jose, CA). Two-tailed p-values $<0.05$ were considered statistically significant.

\section{Results}

The records of 113 patients treated during the six-month study period indicated SB. We excluded 11 patients from that original group: four because the measured band level was missing from the record, and seven because they were over 19 years of age; therefore, 102 patients were included in the final analysis. Mean (SD) was $5.25(0.5)$ years.

Twenty-one patients (21\%) had SAEs, 18 (18\%) had MAEs, 39 (38\%) AAE (Table 1), and 63 (62\%) patients did not have AAE. 


\section{Cureus}

\section{Characteristics}

\section{Results}

Total patients, $\mathbf{N}(\%)$

$102(100)$

Age (years), mean (SD)

Age groups, $\mathrm{N}(\%)$

Two months - two years

Three years -18 years

Gender, N (\%)

Male

Female

Any non-normal physical findings, $\mathrm{N}(\%)$

Fever, $\mathbf{N}(\%)$

WBC (cell/mL), mean (SD)

$12(0.6)$

Bands, mean (SD)

Non-infectious diagnoses, $\mathrm{N}(\%)$

Positive urinalysis result, $\mathrm{N}(\%)$

7 (7)

Positive cultures, N (\%)

Blood culture

CSF

Stool culture

Urine culture

Imaging studies, N (\%)

Non-normal XR

Non-normal CT

$8(8)$

Adverse events, N (\%)

Any adverse event

Moderate adverse event

Severe adverse event

Outcomes, N (\%)

Admission from ED

Return to ED within seven days

Death

$0(0)$

\section{TABLE 1: Characteristics of pediatric patients with severe bandemia.}

Bands were counted as percentage of total WBC count.

Abbreviations: CSF, cerebrospinal fluid; CT, computerized tomography scan; ED, emergency department; $\mathrm{mL}$, milliliters; SD, standard deviation; WBC, white blood cell; XR, X-ray radiograph

Among the 21 SAE patients, 17 were admitted during the index ED visit and four were discharged during the initial visit but returned to the ED within seven days. There was no mortality and there was no readmission within seven days after the index ED visit. The most common diagnosis for 39 patients with AAE was pneumonia (10\%), fever (5\%), and appendicitis (4\%) (Figure 1). 


\section{Cureus}

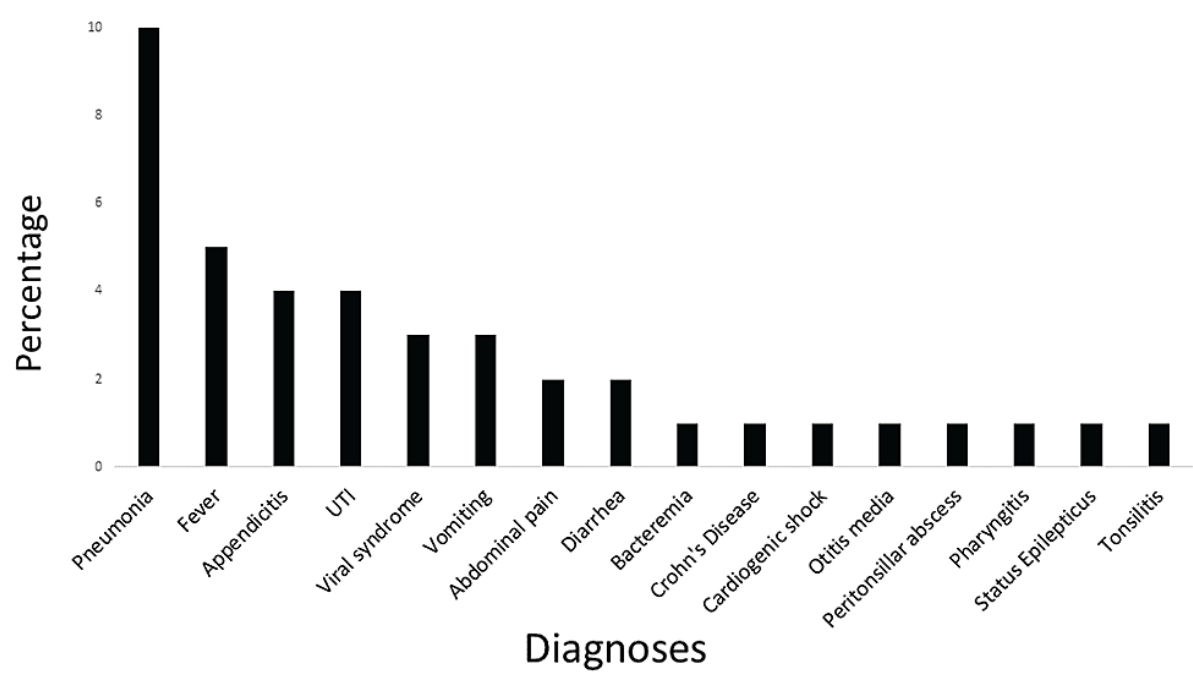

FIGURE 1: Hospital diagnoses of patients who had any adverse events.

Abbreviations: UTI, urinary tract infection

Characteristics of patients between groups were similar (Table 2). Mean band level for patients with no adverse events was 28 (10). This level was non-statistically similar (p-value=0.64) to patients with SAE, MAE, or AAE with mean band levels of 31 (9), 27 (8), and 29 (9), respectively. Patients with no adverse events had significantly less non-normal XR and CT findings $(\mathrm{p}<0.001)$, compared to those with adverse events.

\begin{tabular}{|c|c|c|c|c|c|}
\hline Variables & No Adverse Events $(\mathrm{N}=63)$ & SAE (N=21) & MAE (N=18) & AAE $(\mathrm{N}=39)$ & $\mathbf{P}$ \\
\hline Age (years), mean (SD) & $5(5)$ & $7(5)$ & 4 (4) & $6(5)$ & 0.14 \\
\hline \multicolumn{6}{|l|}{ Gender, N (\%) } \\
\hline Female & 31 (49) & $14(66)$ & $7(39)$ & 21 (54) & \multirow[t]{2}{*}{0.35} \\
\hline Male & $32(50)$ & $7(33)$ & $11(61)$ & $18(46)$ & \\
\hline Non-normal PE findings, N (\%) & $29(46)$ & $13(62)$ & $5(28)$ & $18(4)$ & 0.21 \\
\hline Fever, N (\%) & $24(48)$ & $5(24)$ & $9(50)$ & $14(36)$ & 0.40 \\
\hline WBC $\left(10^{3}\right.$ cell $\left./ \mathrm{mL}\right)$, mean (SD) & $11(5)$ & $13(8)$ & $14(6)$ & $14(7)$ & 0.07 \\
\hline Bands*, mean (SD) & $28(10)$ & $31(9)$ & $27(8)$ & $29(9)$ & 0.64 \\
\hline Non-normal XR finding, N (\%) & $1(2)$ & $3(14)$ & $8(44)$ & $11(28)$ & $<0.001$ \\
\hline Non-normal CT finding, N (\%) & $1(2)$ & $6(28)$ & $1(5)$ & $7(18)$ & 0.002 \\
\hline
\end{tabular}

\section{TABLE 2: Characteristics of patients having adverse events.}

*Bands were defined as percentage of total WBC count.

Abbreviations: $\mathrm{AAE}$, any adverse event; $\mathrm{CT}$, computerized tomography scan; $\mathrm{mL}$, milliliters; $\mathrm{MAE}$, moderate adverse event; $\mathrm{PE}$, physical examination; SAE, severe adverse event; SD, standard deviation; WBC, white blood cell; XR, X-ray radiograph 


\begin{tabular}{|c|c|c|c|c|c|c|c|c|c|}
\hline \multirow[t]{2}{*}{ Varrables } & \multicolumn{3}{|l|}{ AAE } & \multicolumn{3}{|l|}{ MAE } & \multicolumn{3}{|l|}{ SAE } \\
\hline & OR & $95 \% \mathrm{Cl}$ & $P$ & OR & $95 \% \mathrm{Cl}$ & $P$ & OR & $95 \% \mathrm{Cl}$ & $P$ \\
\hline Age (years) & 0.97 & $0.8-1.1$ & 0.62 & 0.97 & $0.8-1.1$ & 0.58 & 1.03 & $0.9-1.2$ & 0.61 \\
\hline Gender & 0.91 & $0.3-2.6$ & 0.85 & 0.91 & $0.2-2.5$ & 0.42 & 0.47 & $0.1-1.5$ & 0.20 \\
\hline Non-normal PE & 0.84 & $0.3-2.4$ & 0.76 & 0.84 & $0.3-2.4$ & 0.16 & 1.15 & $0.4-3.7$ & 0.82 \\
\hline Fever & 0.68 & $0.2-2.2$ & 0.52 & 0.68 & $0.2-2.2$ & 0.92 & 0.84 & $0.2-3.1$ & 0.80 \\
\hline WBC & 1.04 & $0.9-1.2$ & 0.38 & 1.01 & $0.9-1.1$ & 0.82 & 1.03 & $0.9-1.1$ & 0.45 \\
\hline Bands & 1.01 & $0.9-1.1$ & 0.49 & 0.97 & $0.9-1.1$ & 0.43 & 1.04 & $0.9-1.1$ & 0.27 \\
\hline Non-normal XR & 32 & 3.5-100+ & $<0.001$ & 17 & $3.3-90$ & $<0.001$ & 1.08 & $0.2-1.2$ & 0.94 \\
\hline Non-normal CT & 27 & $2.5-100+$ & 0.001 & 1.2 & $0.1-14$ & 0.91 & 15.4 & $2.2-100+$ & 0.002 \\
\hline
\end{tabular}

\section{TABLE 3: Results from multivariate logistic regressions.}

Abbreviations: $\mathrm{AAE}$, any adverse event; $\mathrm{Cl}$, confidence interval; $\mathrm{CT}$, computerized tomography scan; MAE, moderate adverse event; OR, odds ratio; $\mathrm{PE}$, physical examination; SAE, severe adverse event; WBC, white blood cell; XR, X-ray radiograph

\section{Discussion}

In our study, based on a general pediatric ED population, SB (band cells constituting more than $20 \%$ of the total peripheral WBC count) was not associated with a higher risk of adverse outcomes caused by bacterial illness. None of the patients in our study group died during hospitalization or required hospital readmission within seven days after their initial ED evaluation. Our observations suggested that SB should not be used as a single factor that influences clinical decisions.

Further studies in pediatric patients are needed to confirm our finding that SB was not associated with clinical outcomes. However, our finding was consistent with previous studies involving adult ED patients. Ward et al. [10] reported that median bandemia levels for septic adult survivors were $9 \%$, compared to $17 \%$ for septic non-survivors ( $\mathrm{p}$-value $=0.766$ ) and increasing band level was not associated with increased odds of death $(95 \%$ CI 0.99-1.028, $\mathrm{p}$-value $=0.354)$.

SB was noted in patients with other inflammatory causes for admission, such as status epilepticus and cardiogenic shock from congenital heart disease, suggesting that bandemia serves as a good inflammatory marker but might not be a good marker for severe infection or adverse clinical outcomes. In contrast, in a study of children with appendicitis [11], Whyte and associates reported that nonoperative management failed more often in those with bandemia greater than $11 \%$ than in those with bandemia less than $10 \%$, which resulted in longer hospital length of stay (LOS) (17 vs. 9 days). The difference between our findings and Whyte et al.'s could be explained by the low prevalence of appendicitis (4\%) in our study population or a low prevalence of SBI among our ED pediatric population with SB.

Four (4\%) patients in the SAE group returned to the ED within seven days after their initial ED visit because of abdominal pain, vomiting, or fever. None were re-admitted to the hospital. This rate of ED return is similar to those documented in previous reports $[12,13]$. Most pediatric patients return to EDs because their disease progresses, and up to $30 \%$ of them are admitted [14]. In our population, returning patients with SAE were not admitted at the second visits, possibly because the sample of returning patients was small (4\%) and because of the poor association between SB and these patients' diseases.

Our study also found no association between SB and a higher risk of MAE caused by bacterial illness. The most common diagnoses for patients in the MAE group were pneumonia (8\%) and urinary tract infection (UTI) (4\%). Patients in this group were discharged with antibiotics and did not return to the ED or get readmitted to the hospital within the subsequent seven days. Thus, without other indicators such as hypoxia, SB did not affect outcomes among pediatric patients with these infections.

\section{Limitations}

Our study has several limitations. In addition to those inherent to retrospective studies, we did not have an age-matched control group with band counts constituting $<20 \%$ of the total peripheral WBC count and thus could not compare the rates of adverse events between the study group and a control group. Therefore, we were not able to calculate sensitivity or specificity of SB as predictors of clinical outcomes. Furthermore, as a single-center study, we might have missed patients who returned to EDs at other hospitals, although this 
missing rate might be low because the study hospital is the only tertiary referral pediatric hospital in the region.

This study provides information that will be helpful in the design of future investigations of bandemia and outcomes among pediatric patients. Future studies with a higher percentage of surgical patients will provide more conclusive information regarding SB in medical vs. surgical patients, such as those with appendicitis, which might be associated with higher levels of bandemia and different outcomes from those of medical pediatric patients.

\section{Conclusions}

SB is an inflammatory marker and is not associated with higher risks of adverse events caused by bacterial illness among ED pediatric patients. SB should not be used as a single factor in clinical decisions. Clinicians should base their clinical judgment on the overall context of physical examination findings, other laboratory data, and imaging studies.

\section{Additional Information}

\section{Disclosures}

Human subjects: Consent was obtained or waived by all participants in this study. Ethics committee at the Inova Fairfax Hospital issued approval not applicable. The study was approved by the ethics committee at the Inova Fairfax Hospital, Falls Church, VA. The decision was to waive any formal consent. Animal subjects: All authors have confirmed that this study did not involve animal subjects or tissue. Conflicts of interest: In compliance with the ICMJE uniform disclosure form, all authors declare the following: Payment/services info: All authors have declared that no financial support was received from any organization for the submitted work. Financial relationships: All authors have declared that they have no financial relationships at present or within the previous three years with any organizations that might have an interest in the submitted work. Other relationships: All authors have declared that there are no other relationships or activities that could appear to have influenced the submitted work.

\section{Acknowledgements}

We thank Dr. Rick Place and Mr. Travis Tracy for their contributions to the manuscript. We also thank Linda J. Kesselring, MS, Editors in the Life Sciences, for copyediting the manuscript.

\section{References}

1. Schuller KA, Hsu BS, Thompson AB: The rate of sepsis in a national pediatric population, 2006 to 2012 . Clin Pediatr. 2017, 56:1001-1007. 10.1177/0009922817702940

2. Nierhaus A, Klatte S, Linssen J, et al.: Revisiting the white blood cell count: immature granulocytes count as a diagnostic marker to discriminate between SIRS and sepsis - a prospective, observational study. BMC Immunol. 2013, 14:8. 10.1186/1471-2172-14-8

3. Hsueh L, Molino J, Mermel L: Elevated bands as a predictor of bloodstream infection and in-hospital mortality (IN PRESS). Am J Emerg Med. 2020, 10.1016/j.ajem.2020.11.049

4. Jaskiewicz JA, Mccarthy CA, Richardson AC, et al.: Febrile infants at low risk for serious bacterial infectionan appraisal of the Rochester criteria and implications for management. Pediatrics. 1994, 94:390-396.

5. Schnadower D, Kuppermann N, Macias CG, et al.: Febrile infants with urinary tract infections at very low risk for adverse events and bacteremia. Pediatrics. 2010, 126:1074-1083. 10.1542/peds.2010-0479

6. Isaacman DJ, Shults J, Gross TK, Davis PH, Harper M: Predictors of bacteremia in febrile children 3 to 36 months of age. Pediatrics. 2000, 106:977-982. 10.1542/peds.106.5.977

7. Kanegaye JT, Nigrovic LE, Malley R, et al.: Diagnostic value of immature neutrophils (bands) in the cerebrospinal fluid of children with cerebrospinal fluid pleocytosis. Pediatrics. 2009, 123:e967-e971. 10.1542/peds.2008-1742

8. Kuppermann N, Walton EA: Immature neutrophils in the blood smears of young febrile children . Arch Pediatr Adolesc Med. 1999, 153:261-266. 10.1001/archpedi.153.3.261

9. Shi E, Vilke GM, Coyne CJ, Oyama LC, Castillo EM: Clinical outcomes of ED patients with bandemia. Am J Emerg Med. 2015, 33:876-881. 10.1016/j.ajem.2015.03.035

10. Ward MJ, Fertel BS, Bonomo JB, Smith CL, Hart KW, Lindsell CJ, Wright SW: The degree of bandemia in septic ED patients does not predict inpatient mortality. Am J Emerg Med. 2012, 30:181-183. 10.1016/j.ajem.2010.08.018

11. Whyte C, Levin T, Harris BH: Early decisions in perforated appendicitis in children: lessons from a study of nonoperative management. J Pediatr Surg. 2008, 43:1459-1463. 10.1016/j.jpedsurg.2007.11.032

12. Berry JG, Toomey SL, Zaslavsky AM, et al.: Pediatric readmission prevalence and variability across hospitals . JAMA. 2013, 309:372-380. 10.1001/jama.2012.188351

13. Bucholz EM, Toomey SL, Schuster MA: Trends in pediatric hospitalizations and readmissions: 2010-2016. Pediatrics. 2019, 143:e20181958. 10.1542/peds.2018-1958

14. Ali AB, Place R, Howell J, Malubay SM: Early pediatric emergency department return visits: a prospective patient-centric assessment. Clin Pediatr. 2012, 51:651-658. 10.1177/0009922812440840 